Jörg W.E. Fassbinder

\title{
Magnetometerprospektion sakischer Kurgane: Das Gräberfeld Zoan Tobe mit einem Großkurgan und der Kurgan bei Kegen, Süd-Ost Kasachstan
}

Keywords: Magnetometerprospektion, Cäsium-Magnetometer, Kurgane, Skythen-/Sakenzeit, Čilik, Kegen, Kasachstan

\section{Einführung}

Die Kooperation des Bayerischen Landesamtes für Denkmalpflege - und seit 20ıo des Instituts für Geophysik der Ludwig-Maximilians-Universität München - mit den Berliner Forschungseinrichtungen auf dem Gebiet der geophysikalischen Prospektion besteht bereits seit Gründung der EurasienAbteilung des Deutschen Archäologischen Institutes im Jahre I995. Gleich zu Beginn dieser Kooperation gelang es mit dem Nachweis großflächiger Siedlungen der späten Bronze- und der jüngeren Eisenzeit bei Čiča (Sibirien) und von Gräberfeldern der Bronzezeit bei Suchanicha, die besonderen Möglichkeiten und die Bedeutung dieser naturwissenschaftlichen Methode für die archäologischen Forschungen aufzuzeigen (Becker/Fassbinder 1999; Fassbinder/Becker I999; 2003; 2009). Magnetometer-Messungen auf den großen Kurganen und den monumentalen Grabhügeln waren zunächst nicht im Fokus der ersten Arbeiten, denn man versprach sich davon keinen weiteren Erkenntnisgewinn für die archäologische Forschung. Die Ausgrabungen von Kurganen zielten nach wie vor vorrangig auf die Untersuchung der Grabkammern sowie die Bergung der reichen Beigaben. Da es bisher keine geophysikalische Methode gibt, mit der es möglich wäre, die zum Teil mehr als Io-2o m unter der Hügelkuppe liegenden Grabkammern im Detail zu erfassen, war das Interesse seitens der Archäologie von russischer Seite an einer Prospektion zunächst auch eher verhalten. Mit den schon fast legendären Grabungserfolgen des DAI in Aržan (Sibirien) und in Čiča unter der Leitung von Hermann Parzinger und Vjačeslav Molodin hat sich aber diese Sichtweise der Archäologen stark verändert (Molodin u.a. I999; 200Ia; 200Ib; Schneeweiß 2007). Das Grabungsziel war nun nicht mehr allein auf die vielfach schon in früherer Zeit beraubten Grabkammern und die Vermehrung von Fundmaterial ausgerichtet, sondern zusätzlich auf das Verständnis der Denkmal- und Kulturlandschaft im Umfeld solcher Bauwerke sowie auf den strukturellen Aufbau der Kurgane. Bei den jüngsten Kampagnen der Kooperationspartner, Stiftung Preußischer Kulturbesitz, Deutsches Archäologisches Institut und Excellenzcluster 264 Topoi (alle Berlin), waren daher die nicht nur die Kurgane, sondern auch ihr Umfeld das Ziel geophysikalischer Prospektion. 


\section{Archäologischer Hintergrund}

Skythen und Sarmaten waren Reiternomaden, die die Eurasische Steppe von Südsibirien über Kasachstan705 und den Kaukasus bis nach Ungarn bevölkerten (Parzinger 2004). Mit ihrer Wanderweidewirtschaft dominierten sie die Landschaft der pontischen Steppe im Zeitraum zwischen roo० und 400 v. Chr. und während der gesamten klassischen Antike. Bis vor kurzem galt die Annahme, dass diese Völker zwar riesige Grabmonumente geschaffen haben, aufgrund ihrer Lebensweise aber keine weiteren Siedlungsspuren hinterlassen hätten. Dieses Bild musste mit der Vermessung und den Ausgrabungen der protourbanen Siedlung Čiča in Sibirien revidiert werden (Becker/Fassbinder I999; Schneeweiß 2007). Die im wahrsten Sinne des Wortes herausragenden Bauten der Reiternomaden sind jedoch die bis zu $35 \mathrm{~m}$ hohen und bis zu $200 \mathrm{~m}$ im Durchmesser messenden Kurgane. Sie waren nicht nur Grabmonumente der Könige und Fürsten, sondern dienten der Bevölkerung auch als kultische Treffpunkte und heilige Stätten (Parzinger 2004).

Semireč'e, das „Land der Sieben Flüsse“, liegt im südöstlichen Kasachstan und hat nicht nur seinen Namen, sondern auch seine Fruchtbarkeit und seinen Reichtum den sieben Flüssen Ili, Karatal, Bien, Aksu, Lepsy, Baskan und Sarkand zu verdanken. In diesem Gebiet finden sich Zehntausende von Grabhügeln, darunter Hunderte monumentaler Kurgane.

Die Konzentration von Menschen und Macht in dieser Region basiert auf den riesigen fruchtbaren Ebenen, die sehr gut zur Weidewirtschaft geeignet sind, aber auch auf reiche Gold-, Zinn- und Kupfervorkommen in den Bergen von Semireče.

Die Kurgane, die etwa in das 5. Jh. v. Chr. datieren, treten nicht nur einzeln auf, sondern oftmals in Gruppen. Je höher ein Grabhügel ausfällt, desto bedeutender und reicher war in der Regel die darin bestattete Person. Diese Prunksucht ist typisch für die frühen Reiternomaden des 5.-3. Jh. v. Chr. Die vielen und reichen Grabbeigaben sind die Ursache dafür, dass die Grabhügel schon im Altertum Ziel von Raubgrabungen waren.

Eine Reihe großer, selbst im Satellitenbild sichtbarer Kurgane findet sich etwa I20 km östlich der alten Hauptstadt Almaty rund I2 km nordöstlich der Provinzstadt Čilik. Die Ebene ist im Süden und Norden von hohen Bergen umgeben und liegt nicht weit von der Nordroute der Seidenstraße entfernt. Das Areal war und ist ideales Weideland, konnte seit früher Zeit aber auch ackerbaulich genutzt werden. Der mächtigste Grabhügel von Žoan Tobe wird begleitet von vier weiteren Kurganen. Er hat eine Höhe von I2 m, einen Durchmesser von I07 $\mathrm{m}$ und ist aus vielen Lehm- und Kieselstein-Schichten aufgebaut. Ein großer Grabungstrichter im Zentrum zeigt, dass der etwa 2500 Jahre alte Hügel schon vor über hundert Jahren Ziel einer Raubgrabung war. Zwei weitere Reihen etwas kleinerer Kurgane befinden sich in ca. $600 \mathrm{~m}$ Entfernung, gehörten aber augenscheinlich zur gleichen Nekropole (siehe Abb. 202).

Die archäologische Fragestellung war mehrschichtig. Sind die Kurgane Teil eines Kultplatzes, stehen sie separat oder gibt es Verbindungen zwischen ihnen? Ca. $40 \mathrm{~m}$ vom großen Kurgan entfernt ist ein Steinpflaster erkennbar, dazwischen finden sich weitere kleinere Bestattungen, die sich zum Teil durch ihre Steinpackung an der Oberfläche zu erkennen geben. Die Frage ist: Datieren diese Gräber in dieselbe Zeit wie der große Kurgan, sind sie jünger oder älter? Die Form dieser Gräber ist unterschiedlich; so erscheinen die einen als kreisrunde Steinpackung, andere sind viereckig. Ähnliches zeigt sich

689 Die den Kasachstan besiedelnden Reiternomaden des I. Jt. v. Chr. bezeichnet man als Saken. 
bei der Reihe der größeren Kurgane. Jeweils ein Hügel scheint eine quadratische Form zu besitzen. Zum Verständnis der Fundstelle ist es deshalb notwendig, auch einen Großteil der Peripherie durch eine großflächige geophysikalische Prospektion abzudecken (Fassbinder/Becker 2003).

\section{Magnetometerprospektion}

Die Magnetometrie ist eine „passive“ geophysikalische Prospektionsmethode. Man misst die statischen Veränderungen des natürlichen Erdmagnetfeldes, die durch archäologische Befunde im Untergrund hervorgerufen werden (Aitken I974; Aspinal et al. 2008; Becker 1995; Fassbinder 2009; Scollar et al. 1990). Daher müssen alle natürlichen, technischen und zeitlichen Variationen des Erdmagnetfeldes durch eine Differenzmessung oder durch geeignete andere Verfahren unterdrückt und ausgeblendet werden. Generell bleibt man daher mit magnetometrischen Untersuchungen auf die technisch ungestörten Bereiche außerhalb moderner Siedlungen beschränkt. Bildgebende Verfahren ermöglichen die Transformation der physikalischen Messdaten in ein sogenanntes Magnetogramm und damit die Visualisierung der Daten. Dieses erleichtert eine geophysikalische bzw. archäologische Interpretation und trägt entscheidend zum Erfolg des Prospektionsverfahrens bei. Ein derartiges Messbild lässt auch kleine und sehr schwach magnetische archäologische Befunde in einem Graustufenbild erkennen und sichtbar machen. Eine differenzierte geophysikalische Analyse auf der Grundlage und Kenntnis der magnetischen Eigenschaften von Böden erlaubt dann u.U. eine sehr umfassende archäologische Interpretation der Resultate.

Für die geophysikalische Prospektion kam hier das Cäsium-Magnetometer der Fa. Scintrex Smartmag SM-4G Special zur Anwendung. Dieses Gerät erlaubt es, die Abweichungen vom erdmagnetischen Totalfeld mit einer Empfindlichkeit von \pm Io, o Picotesla bis zu Io Mal pro Sekunde aufzeichnen. Zum Vergleich: Die Stärke des Erdmagnetfeldes, also die Kraft, mit der eine Kompassnadel in die Nordrichtung gezwungen wird, betrug auf den Messflächen der verschiedenen Einsatzgebiete im Mittel etwa 55000 +/-30 Nanotesla und 54330 +/- 30 Nanotesla. Für die Prospektion unterteilt man das Areal in Quadrate von $40 \times 40 \mathrm{~m}$ Die einzelnen Messprofile eines Quadrats werden im Abstand von $50 \mathrm{~cm}$ mit den Cäsium-Sonden (montiert auf einem hölzernen Tragestativ) entlang von Messleinen abgeschritten. Während der Messungen trägt man die Sonden in möglichst konstanter Höhe (ca. $30 \mathrm{~cm}$ ) über dem Boden (siehe Abb. 274). Bei jedem fünften Meter setzt man mittels Handschalter elektronische Markierungspunkte. Interpoliert man die Werte zwischen den Markierungen, so lässt sich rechnerisch über die Geschwindigkeit auch die exakte Position des Messpunktes ermitteln. Je nach individueller Schrittgeschwindigkeit und Messpunktdichte von ca. 6-10 ×50 cm, ergeben sich dann diskrete Werte, die anschließend auf $25 \times 25 \mathrm{~cm}$ interpoliert werden. Die Daten werden als Binärdaten auf der Ausleseeinheit gespeichert, später auf ein Notebook übertragen und mit Hilfe von Bildverarbeitungsprogrammen wie Geoplot (Fa. Geoscan) und Surfer (Fa. Golden Software) als Graustufenbild visualisiert.

Die Anwendung der Cäsium-Magnetometer, sogenannte „Totalfeldmagnetometer“, in dieser speziellen tragbaren Sonden- und Stativorientierung bieten gegenüber den herkömmlichen und weit verbreiteten „Vektor-Magnetometern“ drei entscheidende Vorteile:

I) Die Intensitäten der Magnetfeldanomalien werden in dieser Variometer-, bzw. Duo-Sensor-Konfiguration in voller Intensität aufgezeichnet.

2) Die ohnehin schon größere Empfindlichkeit dieses Magnetometertyps erlaubt es, Strukturen in bis zu 2-3 m Tiefe zu erfassen. Zugleich bleibt der äußere magnetische Störeinfluss auf ein Minimum reduziert, sodass auch die unkompensierten Magnetometerdaten auswertbar sind. 
3) Totalfeldmagnetometer sind relativ tolerant gegenüber einer Verkippung der Messsonden bei Hang-Neigungen bis $\mathrm{zu} \pm 30^{\circ}$.

Alles dies trägt dazu bei, dass wir im Ergebnis insbesondere auf unwegsamen und steilem Gelände, wie sie die Oberflächen der Kurgane darstellen, im Vergleich zu allen herkömmlichen Gerätetypen eine sehr einheitliche, plastische und gut interpretierbare Darstellung der archäologischen Befunde erreichen und zugleich die ohnehin schon ca. Io-fach höhere Empfindlichkeit unseres Magnetometertyps maximal ausnutzen (Fassbinder 2009; Fassbinder/Gorka 2009). In unserer Auswertung werden positive Werte schwarz dargestellt und zeigen die erhöhte Magnetisierung bzw. eine Anreicherung magnetischer Minerale in archäologischen Schichten und Strukturen an (Fassbinder I994).

\section{Resultate}

Im Rahmen zweier Messkampagnen jeweils im Sommer 2008 und Frühjahr 2009 konnten zwei Großkurgane sowie mehrere kleinere Grabhügel bei Čilik und nahe der Provinzhauptstadt Kegen großflächig vermessen werden.

\section{Kurgan 1 der Nekropole Žoan Tobe}

Der große Kurgan I des Gräberfeldes Žoan Tobe (Abb. 202) liegt im Nordosten der Nekropole und dominiert diese Anlage. Die Nekropole besteht aus drei „Ketten“ mehr oder weniger nordsüdlich verlaufender Kurganreihen. Aufgrund der Höhe des Grabhügels waren genaue Erkenntnisse zu Lage und Zustand der Grabkammer nicht zu erwarten. Die Messungen galten daher dem näheren Umfeld sowie der äußeren Struktur und dem Aufbau der Grabanlage. Das Ergebnis zeigt im Magnetbild trotz intensiver zentraler Raubgrabung eine perfekte kreisrunde Form des Monuments. Der kreisrunde zentrale Raubtrichter belegt die effektive und optimierte Arbeitsweise der Grabräuber. Der gesamte Hügel mit seinem Durchmesser von $107 \mathrm{~m}$ bildet sich durch die Vermessung mit dem Totalfeldmagnetometer als ein einziger großer "Störkörper“ ab, dessen Gesamtanomalie aufgrund der Inklination des Erdmagnetfeldes (hier etwa $63^{\circ}$ ) im Süden eine erhöhte und im Norden eine etwas verminderte Intensität aufweist (Abb. 275). Durch die Anwendung eines Hochpassfilters verliert man diese Information, erhält jedoch weitere Details aus den oberflächennahen Schichten der Anlage (Abb. 276). Der Hügel selbst, wie auch das gesamte Areal, ist mit einer Vielzahl kleiner Magnetfeldanomalien belegt, die erst durch die Ergebnisse der Kappa-Messungen (Abb. 277) verständlich und interpretierbar werden. Diese Anomalien zeigen die hohe magnetische Suszeptibilität und die remanente Magnetisierung einzelner Kieselsteine, mit denen der gesamte Hügel belegt ist, an. Der ganze Grabhügel ist also regelhaft und mit mindestens einer Schicht dieser Steine bedeckt. Weitere Strukturen im Aufbau sowie ein tieferer „Einblick“ in die Architektur des Monuments werden dadurch jedoch überdeckt.

Durch das Magnetbild wird darüber hinaus eine $5 \mathrm{~m}$ breite, mit Kieselsteinen geformte Ringstraße sichtbar, die dem Messbild ein dem Planeten Saturn mit seinen Ringen vergleichbares Aussehen verleiht. Diese Ringstraße besteht aus einem Pflaster von etwa IO-30 cm großen Kieselsteinen und weist einen Durchmesser von I87 m auf. Zwischen dieser Pflasterstraße und dem Grabhügel befindet sich eine Vielzahl - insgesamt ca. 25 - weiterer kleinerer Bestattungen und Grabhügel von teils runder, teils 
rechteckiger Form und unterschiedlicher Orientierung. Diese Befunde sprechen für eine lange Nutzung des Areals als Bestattungsplatz.

Modern gestört ist das Messbild lediglich durch eine Vielzahl an von Süd nach Nord verlaufenden Bewässerungsgräben, die sich sehr deutlich (weil oberflächennah) und als helle, „negative“ Signatur darstellen. Der offene noch bestehende Graben bildet sich hier negativ ab, da der Boden ausgehoben wurde und/oder da Wasser diamagnetische Eigenschaften besitzt.

\section{Mittlere Kurgan-Reihe K4 - K7 mit der „Pyramide“ von Žoan Tobe}

Eine weitere Kurganreihe liegt etwa 500 m westlich der Reihe mit dem großen Kurgan I. Sie besteht aus mindestens vier Hügeln, die jedoch wesentlich kleiner als die beiden Kurgane I und 2 sind. Hier konnten die Kurgane 4-6 vermessen werden. Das Messbild (Abb. 278) entstand aus einer geeigneten Überlagerung der Totalfeldmessung mit dem Messbild des Hochpass-Filters, sodass sich alle wesentlichen Elemente und Resultate optimal zu erkennen geben (siehe Abb. 278).

Der nördliche Kurgan 4 ist mit einer Höhe von I, 6 m erhalten, die beiden Kugane 5 und 6 weisen eine Höhe von $4 \mathrm{~m}$ bzw. 3,7 m auf. Sie besitzen Durchmesser von ca. 25 und $40 \mathrm{~m}$ bzw. Seitenlängen von ca. $50 \times 50 \mathrm{~m}$ und bieten daher durchaus geeignete Voraussetzungen, die es erlauben, mit der Magnetometermessung in die Grabkammern zu blicken. So geben sich in allen dreien sowohl die ehemalige Lage der Grabkammer als auch der Aufbau des Grabhügels zu erkennen. Leider wurden auch hier alle Hügel beraubt. Wie bei Kurgan I besteht eine Lage des Grabhügels aus großen Kieselsteinen mit erhöhter magnetischer Suszeptibilität und remanenter Magnetisierung. Weitere kleine Befunde zeichnen sich im näheren Umfeld der Kurgane ab, lassen sich aber aufgrund der starken modernen Störungen durch Pflugspuren und Bewässerungsgräben nur noch sehr vage erkennen.

Im Süden von Grabhügel 5 sowie im Nordwesten des Grabhügels 6 findet sich eine starke und unförmige regellose Anomalie. Sie ist mit hoher Wahrscheinlichkeit auf eine

Blitzschlagmagnetisierung zurückzuführen (Maki 2005; Fassbinder/Gorka 2009). Darauf deutet an dieser Stelle die starke remanente Magnetisierung des Bodens hin, deren Magnetisierungsrichtung uneinheitlich und nicht parallel zum heutigen Erdmagnetfeld ausgerichtet ist. Zu vermuten wäre, dass sich an dieser Stelle einstmals ein hoher Pfosten befunden hat, der dann als „Blitzableiter“ gewirkt hat.

Der Kurgan 6 zeigt im Magnetogramm trotz massiver Beraubung eine nahezu perfekte pyramidale Form mit quadratischem Grundriss und Seitenlängen von ca. $50 \times 50 \mathrm{~m}$. Das Messbild lässt in diesem jedoch noch weitere Details zum Aufbau und zur Konstruktion des Monuments erkennen. Die vier Seitenflächen sind wieder mit Steinen unterschiedlicher magnetischer Suszeptibilität, remanenter Magnetisierung und Magnetisierungsrichtung abgedeckt, deren Strukturen auch aus den Kappa-Messungen an Gesteinsproben sowie aus Proben am Kurgan von Kegen zu erklären sind (siehe dazu auch Abb. 277). Die Kanten und Grenzen der vier Seitenflächen zeichnen sich deutlich durch eine negative Anomalie ab. Ob die Kanten aus Lehm(-ziegeln?) oder aus einer Holzkonstruktion bestanden, lässt sich aus dem Messbild alleine nicht erschließen. Das südliche Trapez weist in zentraler Lage eine Unterbrechung der Steinlage auf und lässt dadurch eine mögliche Nach- oder Nebenbestattung erkennen. Unmittelbar angrenzend an der Nord- und Südseite des Kurgans zeichnen sich zentral eine bzw. zwei weitere Bestattungen ab. Einzelne Anomalien (Gräber oder Gruben) sind regellos auf dem gesamten umgebenden Areal um den Kurgan zu finden. 


\section{Zwei kleine Grabhügel 8 und 9 der dritten Kurgan-Reihe von Žoan Tobe}

Wie schon bei der mittleren Kurganreihe waren die Ackeroberfläche sowie das Gelände um diese zwei Grabhügel in einem für die hochauflösende Magnetometerprospektion denkbar ungünstigen Zustand. Das Gelände war im Frühjahr mit grobem Gerät gepflügt worden, und obgleich der Raps und die Sonnenblumen im Juli bereits geerntet worden waren, blieben die tiefen Ackerfurchen immer noch stark und tief ausgeprägt und erschwerten zusammen mit den hohen Strünken die Prospektionsarbeit und verhinderten ein optimales Ergebnis. Unter normalen Bedingungen wäre eine Prospektion auf diesem Areal abzulehnen.

Das Resultat zeigt zwei verschiedene Grabhügel (Abb. 279). Der nördliche Hügel ist noch in einer Höhe von I,2 m erhalten, er weist einen quadratischen Grundriss von ca. I8 × I8 m auf, die Strukturen liegen dezentral im Südwesten des Quadrates und bestehen eventuell aus zwei Befunden von je $3 \times 4 \mathrm{~m}$. Weitere Strukturen innerhalb des Hügels könnten auch auf Inhomogenitäten der Überdeckung zurückzuführen sein.

Der südliche Grabhügel (mit einer Höhe von etwa I,2 m sowie einem Durchmesser von ca. 2I m) umschließt einen Kreis von etwa I4 $\mathrm{m}$ und zeigt in seinem Inneren ebenfalls zwei Gruben bzw. Strukturen von ca. $3 \times 4 \mathrm{~m}$ Größe. Die Strukturen im Umfeld der beiden Hügel sind wieder stark durch die moderne Feldbearbeitung gestört und lassen sich nicht genauer interpretieren.

\section{Der große Kurgan von Kegen}

Der Kurgan von Kegen liegt etwa $3 \mathrm{~km}$ südöstlich der Ortschaft Kegen. Ein Satellitenbild zeigt ein sehr ungewöhnliches Bild des Hügels, das verständlicherweise zunächst größtes Interesse bei den Archäologen erregte (Abb. 280). Nicht zuletzt deshalb wurde eine Magnetometerprospektion des gesamten Geländes durchgeführt, um die Strukturen und den Aufbau dieses wie von Sonnenstrahlen umgebenen Kurgans zu klären.

Vor Ort ist dieser Satellitenbildbefund kaum noch nachzuvollziehen; es zeigte sich jedoch, dass die hellen Partien des Satellitenbildes kleine Erhebungen im Gelände darstellen, die als gute Reflektoren wirkten und das Bild an dieser Stelle aufhellen.

Durch eine großflächige Magnetometerprospektion konnte das Phänomen danach gänzlich erklärt werden (Abb. 28I-282). Im Magnetbild sind diese strahlenförmigen Strukturen als schwache und vage Anomalie zu identifizieren. Das belegt, dass wir es hier mit einem sogenannten „negativen“ Baubefund zu tun haben. Offenbar wurde zum Bau des Kurgans Erdreich aus den Segmenten zwischen den „Strahlen“ abgetragen und zur Aufschüttung des Hügels verwendet. Die jeweiligen Stege dienten als Transportwege und blieben erhalten. Sie bestehen deshalb wie die Geoglyphen und Linien von Nasca aus verdichtetem Boden und bilden sich nun im Satellitenbild als guter Reflektor ab.

Das gesamte Umfeld des Kurgans, insbesondere die Ränder des Monuments, sind leider auch hier bereits sehr stark durch die landwirtschaftliche Nutzung in Mitleidenschaft gezogen und gestört. Auch dieser Kurgan ist beraubt. Dabei hat man von Osten her das Zentrum mit der vermuteten Grabkammer angegraben.

Das Magnetogramm gibt den beklagenswerten Zustand des Grabhügels zu erkennen (Abb. 28I). So zeigt sich nicht nur die starke Zerstörung durch den Grabraub der letzten Jahrhunderte, bei dem im Osten große Teile des Hügels abgegraben wurden. Im Zentrum steht darüber hinaus ein topographi- 
scher Messpunkt, dessen Eisenkonstruktion das Magnetbild an dieser Stelle zusätzlich verunklart. Aber auch an den Rändern des ehemals wohl etwa I80 $\mathrm{m}$ im Durchmesser messenden Hügels werden die erheblichen Zerstörungen durch den modernen Ackerbau sichtbar. Einen sehr interessanten Einblick in den Aufbau des Kurgans gibt das Messbild dennoch zu erkennen. So war die Oberfläche des Kurgans wohl in einzelne dreieckige Segmente unterteilt, die wechselweise mit Kieselsteinen (von ca. Io-30 cm Größe) und mit homogenen Lehm gepflastert bzw. bedeckt waren. Die Steinbedeckungen zeichnen sich dabei im Magnetogramm jeweils durch eine Vielzahl kleiner unregelmäßiger Anomalien ab, die sich auf die unterschiedliche Suszeptibilität und Magnetisierung der Steine zurückführen lassen (siehe Abb. 277). Die Lehmbedeckung bildet sich demgegenüber eher gleichförmig und homogen ab. Das Magnetogramm auf dem Kurgan selbst gibt daher, wie zu erwarten, keinen Einblick in die zentrale Grabkammer, lässt aber sehr gut das Bauschema der äußersten Schicht erkennen, das sich ähnlich einer unregelmäßig geschnittenen Torte mit unterschiedlichem Belag darstellt.

Im Süden des Hügels findet sich eine Struktur, die analog zum quadratischen Kurgan von Žoan Tobe eine Neben- oder Nachbestattung anzeigen könnte. Im weiteren Umfeld dieses Kurgans sind lediglich ein paar formlose uneinheitliche Grubenkomplexe zu erkennen, die aber nicht als klare Grabstrukturen zu identifizieren sind.

\section{Zusammenfassung}

Die geophysikalische Prospektion ganzer Denkmalgattungen sowie die Magnetometerprospektion von Grabhügeln und Kurganen stellt ein langjähriges Forschungsziel unserer Arbeitsgruppe dar (Fassbinder et al. 2009; 20I3; Gorka/Fassbinder 20II; Molodin et al. 2010). Damit ergibt sich nicht nur die Chance, durch Vergleich der Ergebnisse die für eine Denkmalgattung typischen Befundmerkmale herauszuarbeiten und damit die Interpretation sowie die Datierung über die Form der Anlagen zu verbessern, sondern zugleich die geophysikalische Prospektionsmethode methodisch weiterzuentwickeln und auf die speziellen Anforderungen zu optimieren.

Im Ergebnis liefert die Geophysik genaue Pläne dieser Monumentalbauwerke der Skythen bzw. Saken. Obgleich es aus physikalischen Gründen unmöglich ist, mit magnetischen Messungen detaillierte Strukturen der Grabkammern in größerer Tiefe zu kartieren, gelingt es doch oft, entscheidende, wertvolle und zusätzliche Informationen zum Aufbau zur Architektur und zur Umgebung der Kurgane zu erhalten, die möglicherweise auch einer guten und umfassenden archäologischen Grabung entgangen wären.

Die Ergebnisse der geophysikalischen Prospektion rücken diese Monumente nicht mehr nur als archäologische Objekte, sondern auch als grandiose Baudwerke ins Bewusstsein, die aus der einzigartigen Steppenlandschaft nicht spurlos verschwinden dürften. 


\section{Literatur}

\section{Aitken 1974}

M. J. Aitken, Physics and Archaeology (Oxford I974).

Aspinall et al. 2008

A. Aspinall/C. F. Gaffney/A. Schmidt, Magnetometry for archaeologists (Lanham/ New York/ Toronto 2008).

\section{Becker 1995}

H. Becker, From Nanotesla to Picotesla - a new window for magnetic prospecting in archaeology. Archaeological Prospection 2, I995, 2I7-228.

\section{Becker/Fassbinder 1999}

H. Becker/J. W. E. Fassbinder, Magnetometry of a Scythian settlement near Čiča in the Baraba Steppe. In: J. W. E. Fassbinder/W. E. Irlinger (eds.), Archaeological Prospection. Arbeitsh. Bayer. Landesamt f. Denkmalpflege ı०8, I999, I68-I72.

\section{Fassbinder 1994}

J. W. E. Fassbinder, Die magnetischen Eigenschaften und die Genese ferrimagnetische Minerale in Böden im Hinblick auf die magnetische Prospektion archäologischer Bodendenkmäler (Buch am Erlach 1994).

\section{Fassbinder 2009}

J. W. E. Fassbinder, Geophysikalische Prospektionsmethoden - Chancen für das archäologische Erbe, in Tocare- Non Tocare. In: E. Emmerling (Hrsg.), ICOMOS. Hefte des Deutschen Nationalkomitees, vol. 47 (München 2009) I0-32.

\section{Fassbinder/Becker 1999}

J. W. E. Fassbinder/H. Becker, Magnetometry of the prehistoric necropolis Suchanicha in the Minusinsk Basin South Siberia. In: J. W. E. Fassbinder/W. E. Irlinger (eds.), Archaeological Prospection. Arbeitsh. Bayer. Landesamt f. Denkmalpflege ı०8, I999, I64-I67.

\section{Fassbinder/Becker 2003}

J. W. E. Fassbinder/H. Becker, Magnetometerprospektion des großen Kurgans I von Bajkara. In: H. Parzinger/V. Zajbert/A. Nagler/A. Plešakov, Der große Kurgan von Bajkara. Studien zu einem skythischen Heiligtum. Archäologie in Eurasien I6 (Mainz am Rhein 2003) I3I-I36.

\section{Fassbinder/Becker 2009}

J. W. E. Fassbinder/H. Becker, Geomagnetische Prospektion. In: H. Parzinger/A. Nagler/N. Leont'ev/ V. Zubkov, Das mehrperiodige Gräberfeld von Suchanicha bei Minusinsk. Zur Stellung der Tagar-Kultur im Rahmen der bronze- und eisenzeitlichen Kulturfrage im Minusinsker Becken. Eurasia Antiqua I5, 2009, 75-79.

\section{Fassbinder/Gorka 2009}

J. W. E. Fassbinder/T. Gorka, Beneath the desert soil archaeological prospecting with caesium magnetometer. In: M. Reindel/ G. A. Wagner (eds), New technologies for archaeology. Multidisciplinary investigations in Palpa and Nasca, Peru (Berlin/ Heidelberg 2009) 49-69.

\section{Fassbinder et. al. 2009}

J. W. E. Fassbinder/T. Gorka/H. Parzinger/A. Nagler, Magnetic prospection of Scythian Kurgans from Čilik, Southeastern Kazakhstan. Revue d'Archéométrie, Suppl. 33, Archeo Sciences 33, 2009, 59-6I.

\section{Fassbinder et. al. 2013}

J. W. E. Fassbinder/T. H. Gorka/A. Nagler/M. A. Chemyakina/ V. I. Molodin, Prospecting of Kurgans by magnetometry: case studies from Kazakhstan, Siberia and North-Caucasus. In: D. Yu. Hookk (edt.), Virtual Archaeology (nondestructive methods of prospections, modeling, reconstructions) (St. Peterburg 20I3) 50-57.

\section{Gorka/Fassbinder $201 \mathrm{I}$}

T. H. Gorka/J. W. E. Fassbinder, Classification and documentation of Kurgans by magnetometry. In: M. Drahor/M. Berge (eds.), Archaeological Prospection (Izmir 20II) I83-I86.

\section{Maki 2005}

D. L. Maki, Lightning strikes and prehistoric ovens: Determining the source of magnetic anomalies using techniques of environmental magnetism. Geoarchaeology 20, 2005, 449-459.

\section{Molodin u.a. 1999}

В. И. Молодин/Г. Парцингер/Х. Бекер/Й. Фассбиндер/М. А. Чемякина/А. Наглер/Р. Нииф/О. И. 
Новикова/А. К. Манштейн/Ю. Н. Гаркуша/А. Е. Гришин/Н. С. Ефремова, Археолого-геофизические исследования российско-германской экспедиции в Барабинской лесостепи. In: Проблемы археологии, этнографии, антропологии Сибири и сопредельных территорий : Материалы VII Годовой итоговой сессии Ин -та археологии и этнографии СО РАН. Вd. V (Новосибирск І999) 454-462.

\section{Molodin u.a. 200Ia}

V. I. Molodin/H. Parzinger/Y. N. Garkusha/J. Schneeweiss/H. Becker/J. Fassbinder/M. A. Chemiakina/A. E. Grishin/O. I. Novikova/N. S. Efremova/A. K. Manstein/P. G. Diadkov/S. K. Vasiliev/L. N. Mylnikova/E. V. Balkov, Archaeological and Geophysical Investigations carried out by the joint Russian-German Expedition at the Bronze/Iron Age settlement of Chicha in the Baraba Steppe. Preliminary results. Archaeology, Ethnology \& Anthropology of Eurasia 3, 200I, I04-I27.

\section{Molodin u.a. 200Ib}

В. И. Молодин/Г. Парцингер /Ю. Н. Гаркуша/ Й. Шнеевайс /А. Е. Гришин/О. И. Новикова/ Н. С. Ефремова /Ж. В. Марченко/Л. Н. Мыльникова /М. А. Чемякина/Е. В. Рыбина, Исследования городища Чича -г в 2оог году. Проблемы архео- логии, этнографии, антропологии Сибири и сопредельных территорий 7, 2001, 382-390.

Molodin et. al. 2010

V. I. Molodin/J. W. E. Fassbinder/T. H. Gorka/ O. A. Pozdnyakova/M. A. Chemyakina/I. A. Durakov/S. Hansen/A. Nagler, Aul-Koschkul-I, a new burrial yard of the ancient Turkic period in the Baraba Steppe: Geophysical Prospection. Archaeology and Ethnography Vestnik Novosibirsk State University 9 (issue 7), 2010, 46-56.

\section{Parzinger 2004}

H. Parzinger, Die Skythen (München 2004).

\section{Schneeweiß 2007}

J. Schneeweiß, Die Siedlung Čiča in der westsibirischen Waldsteppe I. Untersuchungen zur spätbronzebis früheisenzeitlichen Keramik, Chronologie und kulturellen Stellung. Archäologie in Eurasien 22 (Mainz am Rhein 2007).

\section{Scollar et al. I990}

I. Scollar/A. Tabbagh/A. Hesse/I. Herzog, Archaeological Prospecting and Remote Sensing (Cambridge I990). 

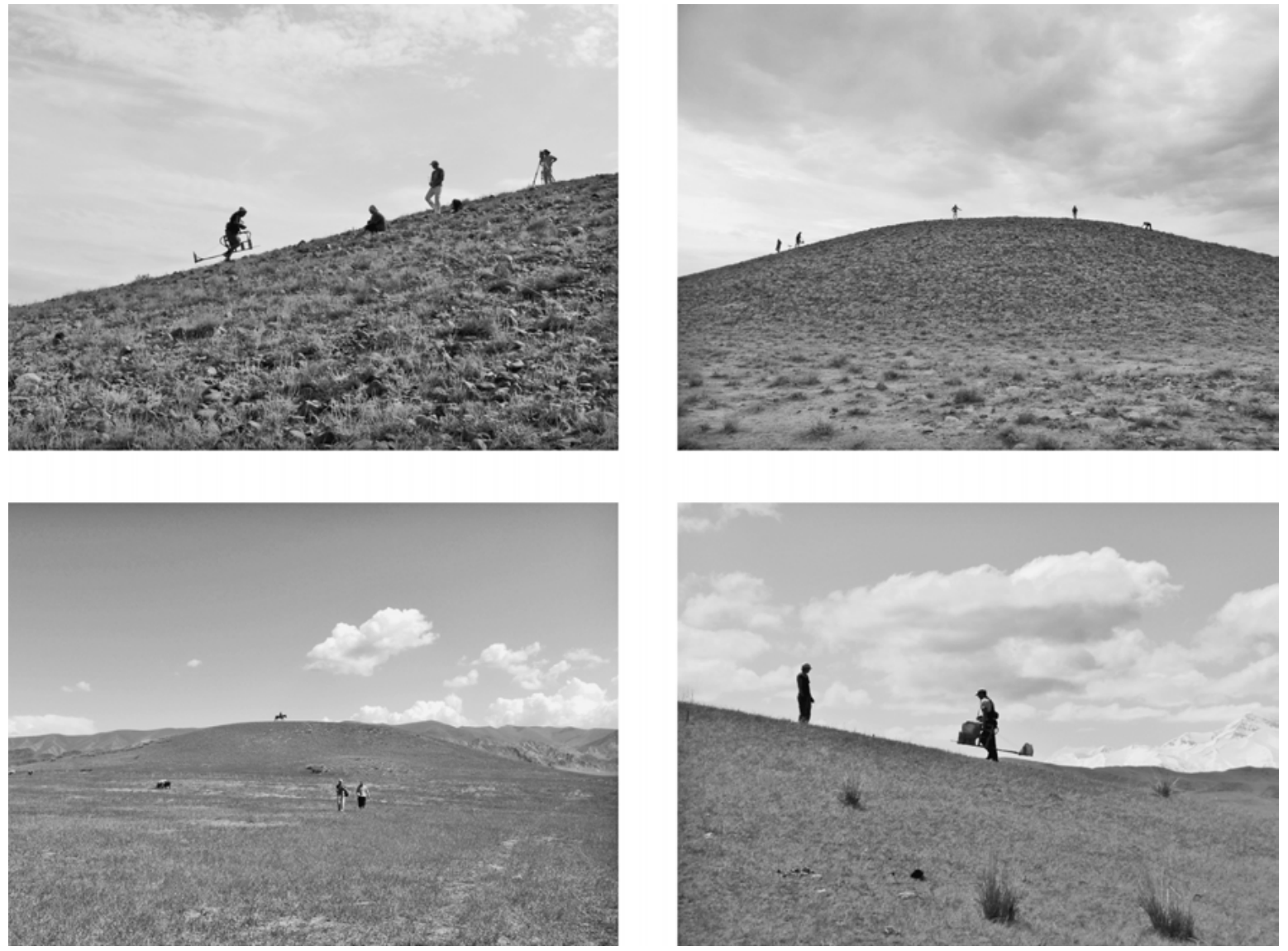

Abb. 274 | Magnetometerprospektion auf den Grabhügeln der Skythen in Čilik und Kegen im Südosten Kasachstans (Foto: Jörg Fassbinder). 


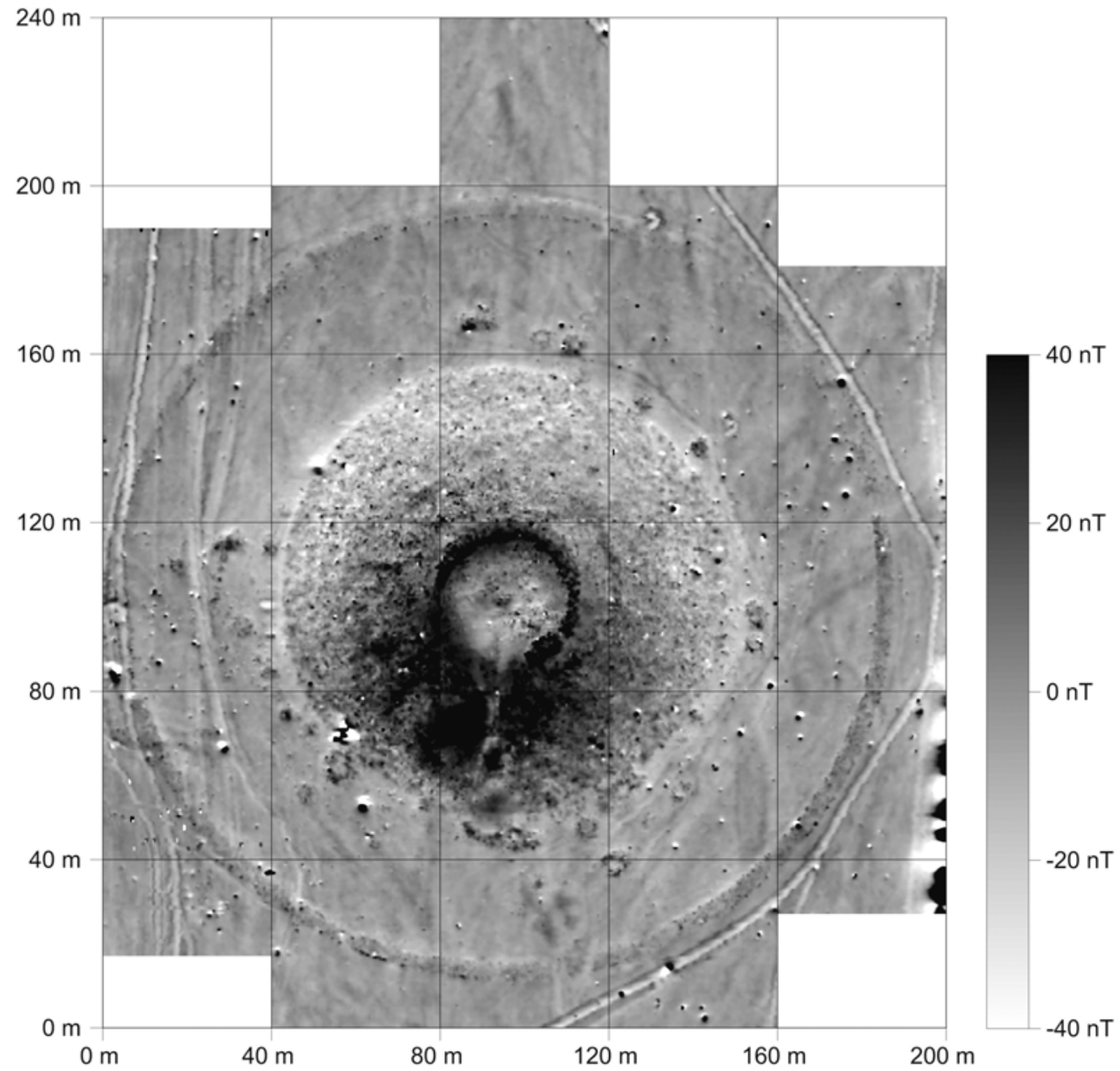

Abb. 275 | Magnetogramm des großen Kurgans I und seines Umfeldes der Nekropole Žoan Tobe bei Čilik. Norden ist oben Magnetogramm: Cäsium-Magnetometer Scintrex Smartmag SM4G-Special, Erdmagnetisches Feld in Čilik im Juni 2008 ca. $55550+/-40$ Nanotesla, Dynamik \pm 40 Nanotesla in 256 Graustufen, Messpunktdichte $50 \times \mathrm{I} 2,5 \mathrm{~cm}$, interpoliert auf $25 \times 25 \mathrm{~cm}$, 40-m-Gitter, Auswertung als Quadratenmittel. (Magnetogramm: J. Fassbinder) 


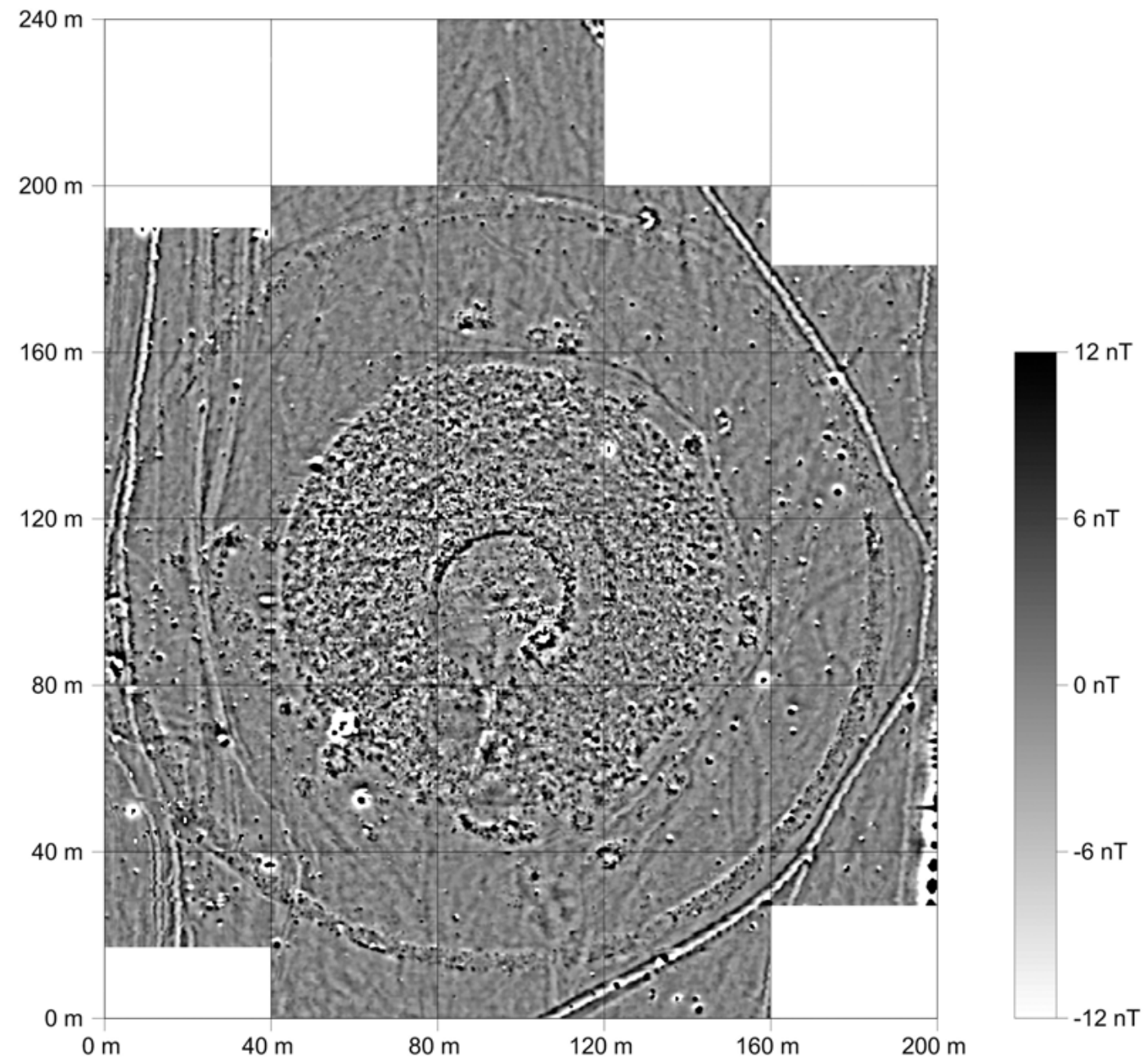

Abb. 276 | Magnetogramm des großen Kurgans I von Žoan Tobe. Technische Daten wie Abb. 275, jedoch mit Anwendung eines Hochpassfilters. (Magnetogramm: J. Fassbinder). 

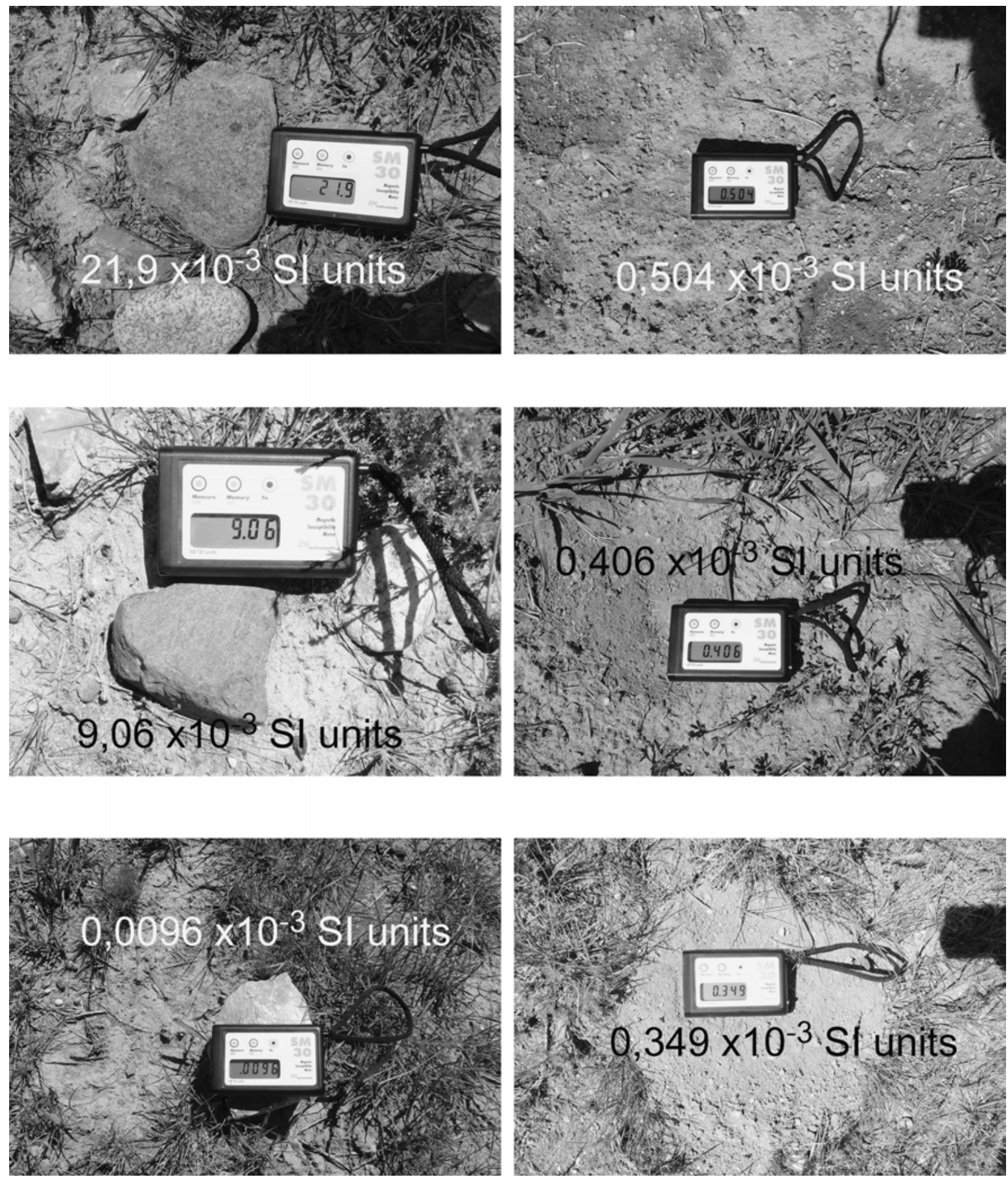

Abb. 277 | Messung der magnetischen Volumen- Suszeptibilität an ausgewählten Gesteinsproben und am Oberboden von Kegen. Kappameter SM 30, Zh-Instruments, Tschechien. 


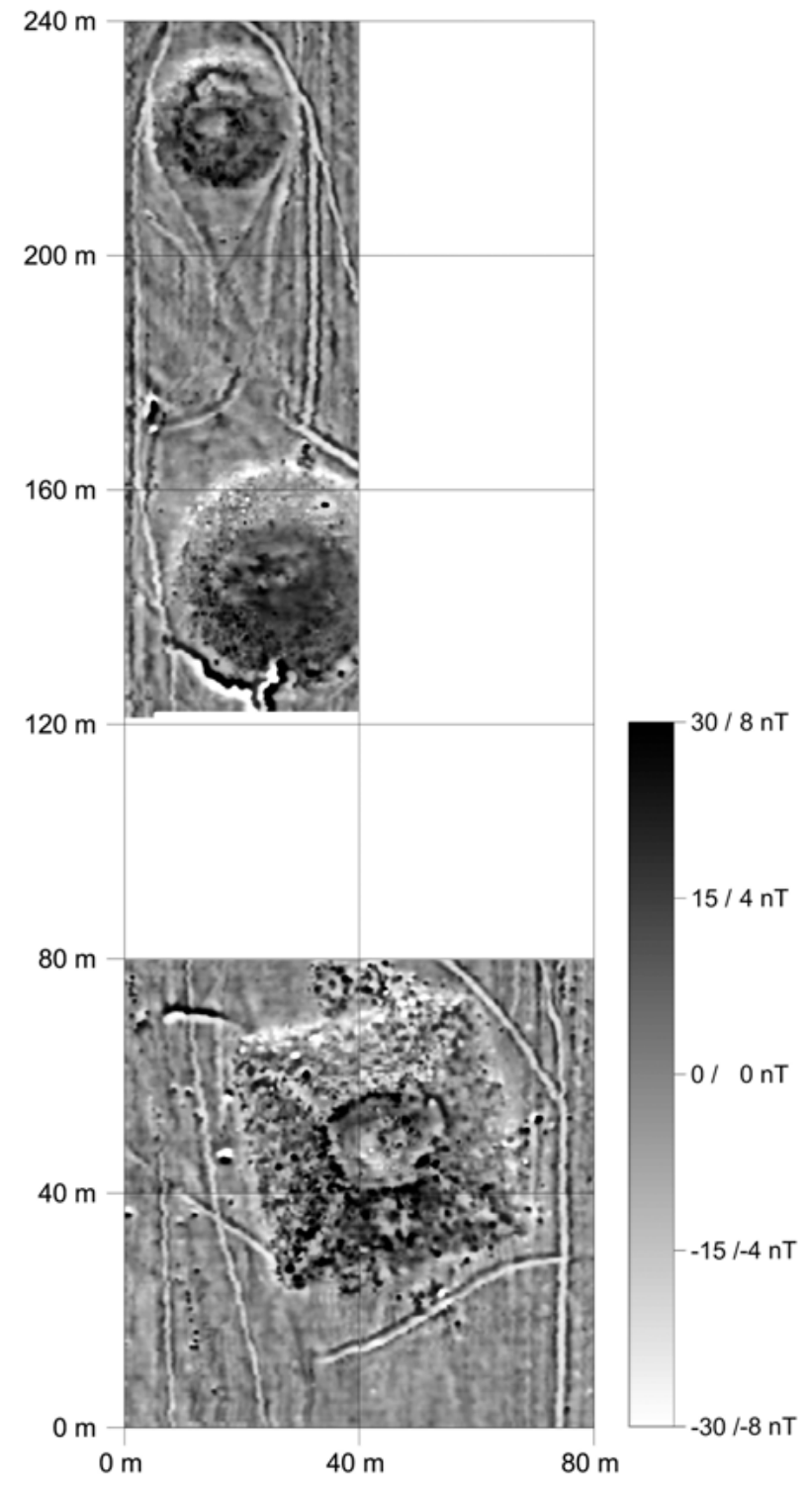

Abb. 278 | Magnetogramm der mittleren Kurganreihe K4-K6 ca. 500 m westlich des Großkurgans I von Žoan Tobe mit seiner Umgebung und dem quadratischen Kurgan 6. Norden ist oben. Magnetogramm: Cäsium-Magnetometer Scintrex Smartmag SM4G-Special, Erdmagnetisches Feld in Čilik im Juni 2008 ca. 55540 +/- 40 Nanotesla, Dynamik \pm 40 Nanotesla in 256 Graustufen, Messpunktdichte $50 \times \mathrm{I} 2,5 \mathrm{~cm}$, interpoliert auf $25 \times 25 \mathrm{~cm}, 40-\mathrm{m}$-Gitter, Auswertung als Quadratenmittel, sowie partiell überlagert durch ein Magnetogram, auf das ein Hochpassfilter angewandt wurde (Magnetogramm: J. Fassbinder). 
Abb. 279 | Magnetogramm der westlichsten Kurganreihe, Kurgane 8 und 9, ca. 700 m entfernt vom großen Kurgan I von Žoan Tobe mit seiner Umgebung. Norden ist oben. Magnetogramm: Technische Daten wie Abb. 278 (Magnetogramm: J. Fassbinder).

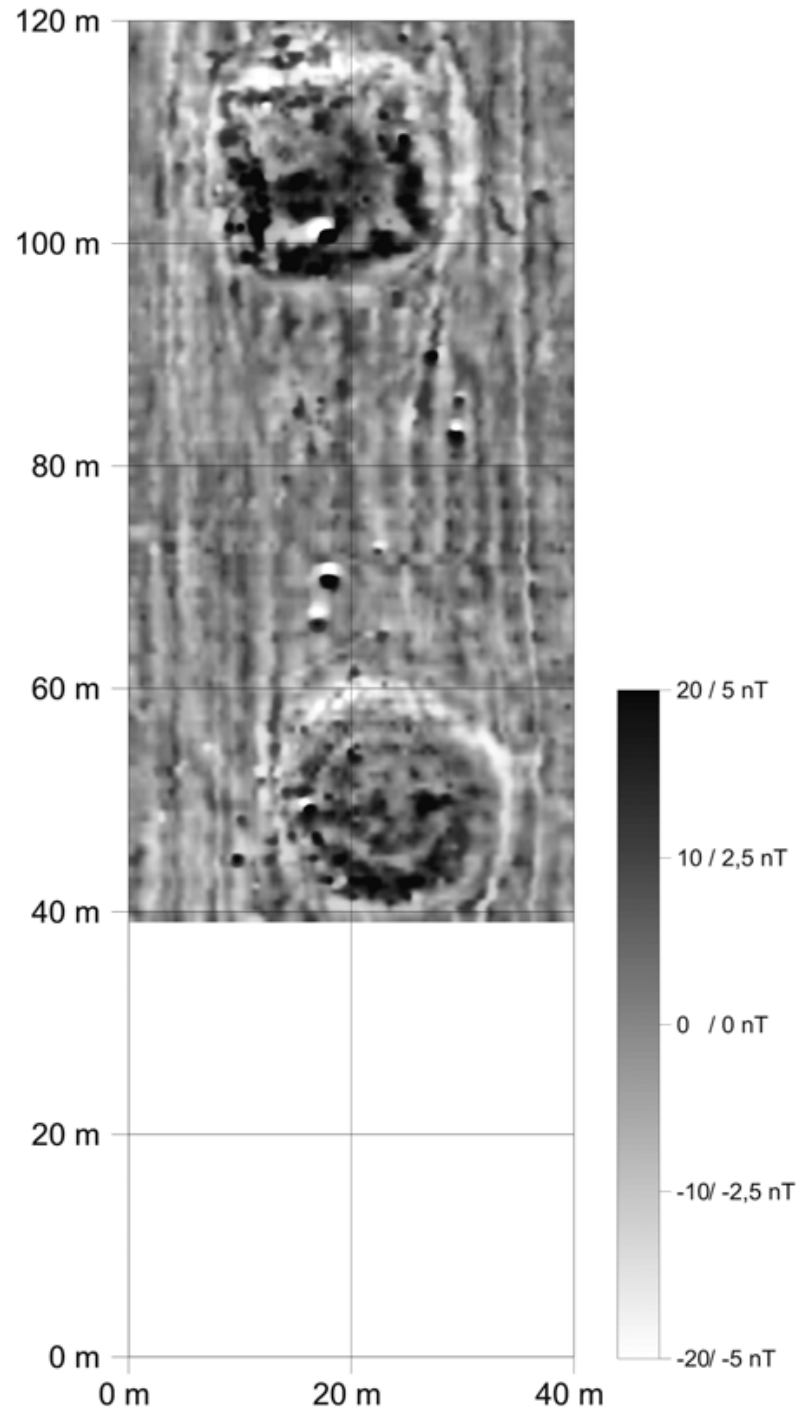




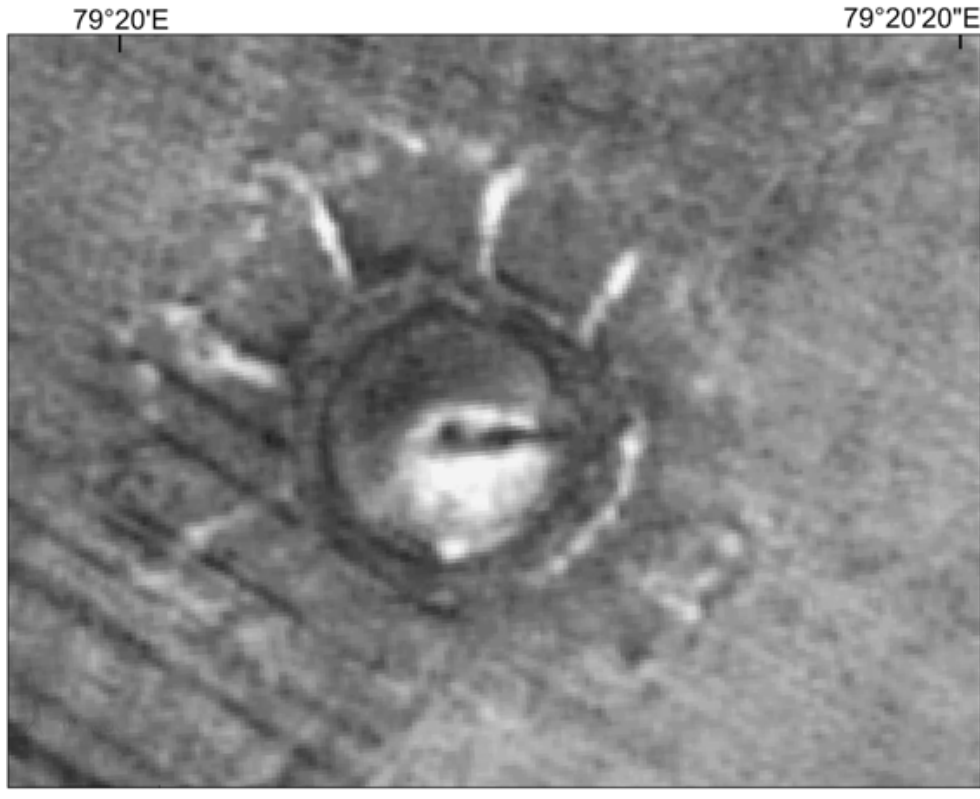

Abb. 280 | Satellitenbild des Kurgans von Kegen. Um den Kurgan werden sternförmige Strukturen sichtbar die sich zunächst aus dem Bild nicht erklären lassen. 


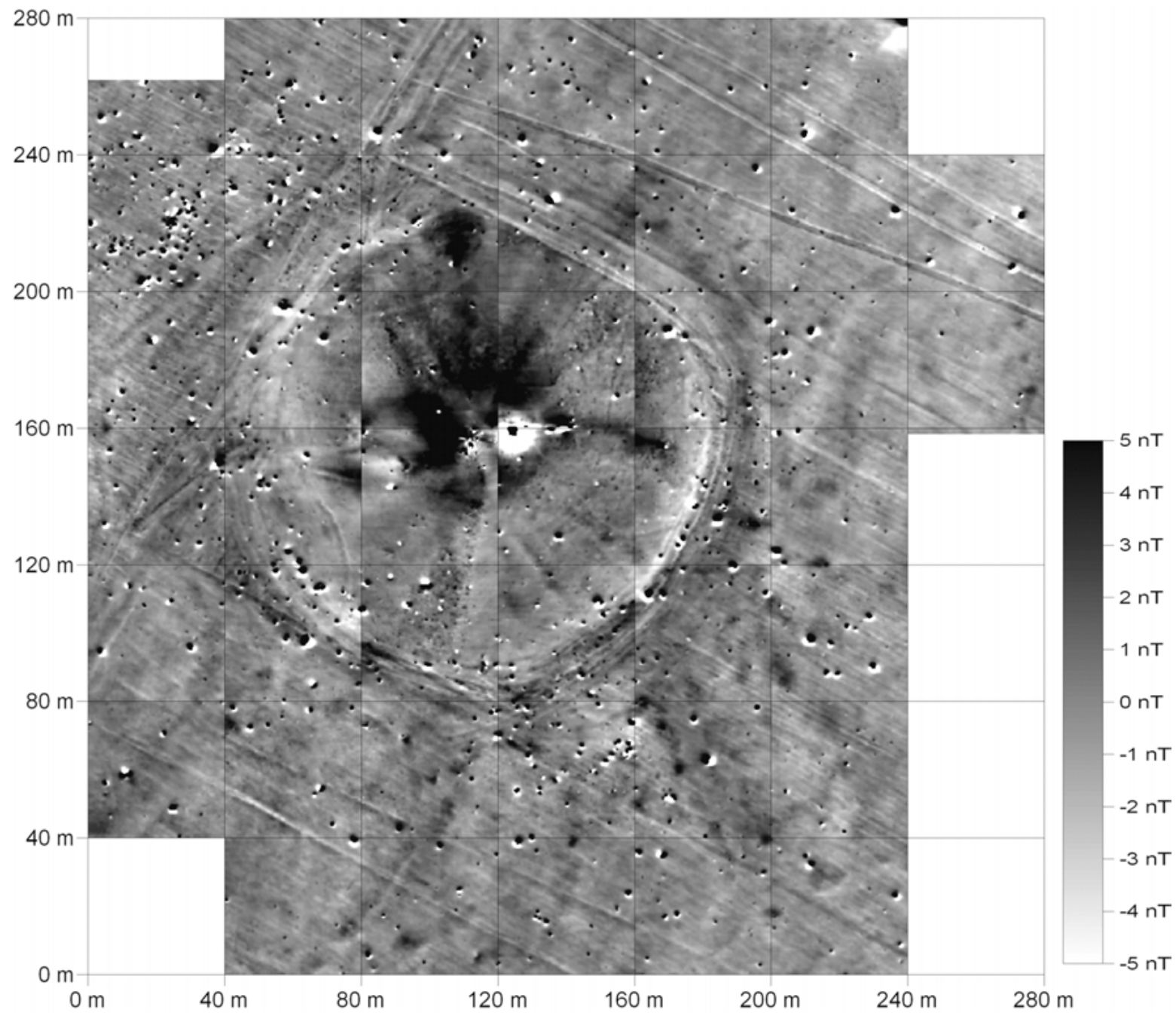

Abb. 281 | Magnetogramm des Kurgans von Kegen. Norden ist unten. Magnetogramm: Cäsium-Magnetometer Scintrex Smartmag SM4G-Special, Erdmagnetisches Feld in Kegen im Juni 2009 ca. 55240 +/- 40 Nanotesla, Dynamik \pm 7 Nanotesla in 256 Graustufen, Messpunktdichte $50 \times 12,5 \mathrm{~cm}$, interpoliert auf $25 \times 25 \mathrm{~cm}, 40-\mathrm{m}$-Gitter, Auswertung als Quadratenmittel.

(Magnetogramm: J. Fassbinder). 


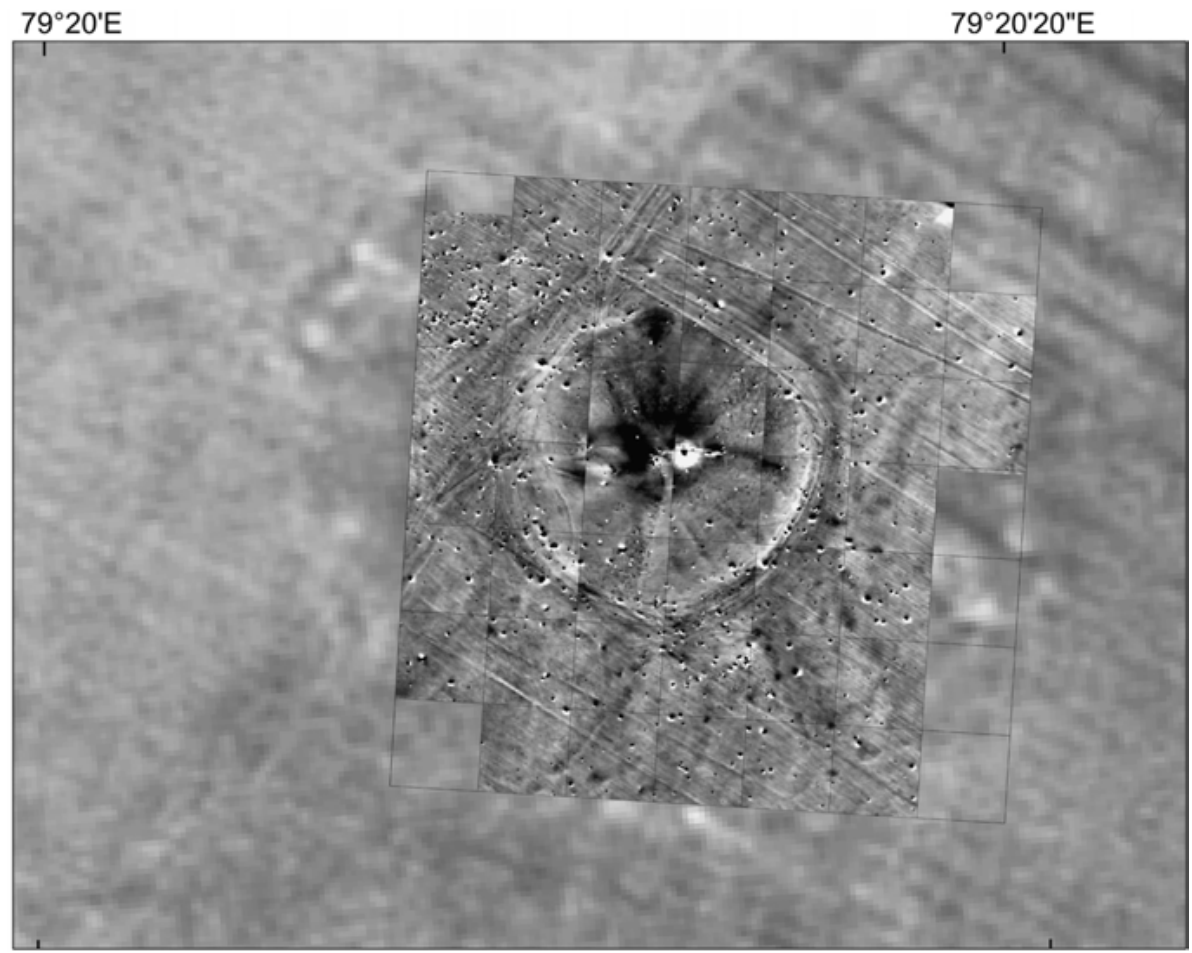

Abb. 282 | Satellitenbild des Kurgans von Kegen. Darin eingeschnitten das Magnetogramm (Technische Daten wie Abb. 28I). 